\title{
Immunoided otological diseases: when to suspect, how to diagnose and treat?
}

\begin{abstract}
The inner ear may be damaged by several autoimmune mechanisms, the most frequent manifestation being progressive sensorineural hearing loss with good responsiveness to immunosuppressants. Eiff work is justified by the need for further studies relating to sensorineural hearing loss mediated (DNSI), since the pathophysiology of this disease entity per obscure manece but positive response to immunosuppressive therapy enhances the existence of immunological mechanisms and autoimmune diseases. The overall objective of this work was to perform a literature review on sensorineural hearing loss immune-mediated-DNSI, focusing on the presentation forms and clinical manifestations, identifying the possible pathophysiological mechanisms involved in internal ear involvement. The specific focus was to establish a protocol to be followed to conduct a suspected case of DNSI, from diagnosis to treatment. The results found in the consulted literature show that the inner ear may be located within the organspecific disease groups, or, more commonly, as a compromised organ within the systemic form, and hearing damage may be the first symptom. The index of inner ear impairment in systemic au toimunes is variable. It can be concluded that the hearing loss is neurossensoria may occur in patients with autoimmune disease and should always be taken in cases of hearing loss without apparent cause and the knowledge of autoimmune diseases and their correlation with the sensorineural hearing loss contributed significantly to the demystification of this type of hearing loss, allowing the institution of specific treatment.
\end{abstract}

Keywords: hearing loss, autoimmunity, sensorineural hearing loss, treatment, otological diseases
Volume II Issue 2 - 2019

\author{
Carlos Eduardo Borges Rezende, Bárbara \\ Teixeira Diony sio, Gabriela Teixeira Diony \\ sio, Li gia Salha Françolin, Nathália Beatriz \\ Pagliarini Martoni \\ Otolaryngologist, Brazil
}

\section{Correspondence: Carlos Eduardo B Rezende,}

Otolaryngologist, CRM 94665, Av Pereira Barreto 1395 Cj 34 North Tower Paradise Sto André, Brazil, Tel 4425 0485, 96403 4192, Email cadurezendel0@gmail.com

Received: June 20, 2017 | Published: March 15, 2019

\section{Introduction}

Autoimmune diseases-DAI are due to an inadequate response of the immune system, which reacts against organs, tissues or cells, leading to their destruction or impairing their function. They include diseases that simultaneously or sequentially affect these organs or systems and others specifically directed to some of them, such as the nervous system, the digestive and respiratory systems, skin, blood, eye , etc. ${ }^{1}$ The immune system is disoriented by attacking the body itself and the organs it should protect and, as a whole, ICDs reach three times as many women as men, and are among the top 10causes of death in women under $65 .^{2}$ Symptoms vary from one disease to another and even within the same disease. Because they are diseases that affect several organs, they may have nonspecific symptoms, which make diagnosis difficult. The same disease may have very different symptoms in several people and at different ages. ${ }^{2}$

Says $^{3}$ that puma autoimmune disease may present as a prolonged fever over three weeks, which does not give with antipyretics or antibiotics, for joint pain with swelling on the red spots on the face that increase with the sun, mouth ulcers that do not pass, miscarriages and repeating, for pericarditis and pleuritis, psychosis, anemia, decreased platelets, eyes and mouth sec the hands that turn white with cold, decreased strength or sensibility in the extremities, kidney failure, hands sweating, tremor es, etc.

These symptoms and signs may be presented $\mathrm{d}$ and slowly, which leads to the patient query, or acute, requiring emergency approach.

Hearing problems can be caused by any disturbance that interferes with the transmission of sound waves from the external environment to the central nervous system. ${ }^{2}$ Deafness is considered sensorineural when the pathology involves structures of the inner ear and auditory nerve. ${ }^{2}$
The determination of the etiology of hearing loss is fundamental for determining prognosis and treatment, which may involve behavioral, clinical, surgical and/or rehabilitation measures with sound amplification. ${ }^{2}$

In the case of ear manifestations, diseases such as sensorineural hearing loss (Table 1) mediated-DNS I having $\mathrm{m}$ was studied and is characterized $\mathrm{m}$ generally by bilateral sensorineural hearing loss, progressive, asymmetric or not accompanied by other symptoms of the inner ear. ${ }^{4}$ It is common for immune-mediated sensorineural hearing loss to be associated with autoimmune systemic diseases, being well characterized in the systemic lupus erythematosus, polyarteritis nodosa, rheumatoid arthritis, giant cell arteritis, Wegener's granulomatosis, ulcerative colitis, Sjögren's syndrome, Behcet's syndrome, amyloidosis, sarcoidosis, Cogan syndrome and Berger's disease. ${ }^{5}$

Immune- mediated sensorineural hearing loss has been studied in the last two decades in relation to its etiological and pathophysiological mechanisms, maintaining itself as a complex disease and difficult to diagnose (Table 2) and treat. ${ }^{2}$ According to ${ }^{2}$ the main clinical features of DNSI are: unilateral or bilateral sensorineural hearing loss that is rapidly progressive or sudden, asymmetric, commonly affecting severe frequencies. In some cases, buzzing and fullness of headset may be present. It can start at any age, being more common in women with age s between 30 and 45years. The diagnosis is eminently clinical and based on the positive response to the therapeutic test with immunosuppressants (Table 3). ${ }^{1}$ The rationale of the effect of immunosuppressants on DAI con focuses on decreasing the efficiency

${ }^{1}$ Immunosuppressants are medicines that lower immunity. They are indicated in the treatment of autoimmune disease, especially when the patient does not respond to conventional medications and are considered the most effective oral remedies. 
of the immune system, passing on the latter to attack the body more mildly, which leads to a decrease in the symptoms of the disease (Table 4).

Hearing loss can be classified in two ways:

a. Sensory: when the inner ear is affected

b. Neural: when there is impairment of the auditory nerve or its complementary pathways. ${ }^{4}$

c. Sensorineural loss, in general, can be caused by viral infections, labyrinthitis, damage to the auditory nerves, exposure to noise, use of some medications or the correlation with diseases such as:

d. Diabetes

Table I Main entities involved in the differential diagnosis of sensorineural hearing loss

\begin{tabular}{|c|c|}
\hline \multicolumn{2}{|r|}{ Characteristics } \\
\hline $\begin{array}{l}\text { Genetic } \\
\text { deafness }\end{array}$ & Autosomal dominant or recessive (most common). \\
\hline Infections & CMV, rubella, toxoplasmosis, gestational or perinatal s) \\
\hline Presbycusis & $\begin{array}{l}\text { Hearing loss associated with aging Intensity and } \\
\text { influenced by: infections, drug use, genetics, exposur } \\
\text { throughout life, comorbidities. }\end{array}$ \\
\hline $\begin{array}{l}\text { Meniérè's } \\
\text { disease }\end{array}$ & $\begin{array}{l}\text { Abnormality of ionic hemostasis and internal ear fluic } \\
\text { endolymph and distension of the membranous labyrin }\end{array}$ \\
\hline Noise-induced & Occupational, use of headphones at high volume Hea \\
\hline $\begin{array}{l}\text { hearing loss } \\
(\mathrm{NIHL})\end{array}$ & $\begin{array}{l}\text { susceptible individuals, if the intensity and time of ex } \\
\text { sufficient. }\end{array}$ \\
\hline Tumors & $\begin{array}{l}\text { Generally benign Most common: acoustic neurinoma } \\
\text { schwannoma). }\end{array}$ \\
\hline $\begin{array}{l}\text { Systemic and } \\
\text { metabolic } \\
\text { changes }\end{array}$ & $\begin{array}{l}\text { Diabetes mellitus, hyperinsulinism, atherosclerosis, } \\
\text { vascular disease, hypertension, dyslipidemia, thyroi } \\
\text { factors possibly associated with hearing loss. }\end{array}$ \\
\hline $\begin{array}{l}\text { Immune- } \\
\text { depleted } \\
\text { deafness }\end{array}$ & $\begin{array}{l}\text { It may be associated with other systemic autoimmur } \\
\text { Substances that may affect the auditory and vestibular }\end{array}$ \\
\hline Ototoxicidade & $\begin{array}{l}\text { Antibiotics and chemotherapeutics: gentamicin, str } \\
\text { tobramycin, cisplatin, amikacin, neomycin, among oth } \\
\text { diuretics (furosemide) and salicylates are also classically }\end{array}$ \\
\hline Neurological & $\begin{array}{l}\text { Stroke (stroke), multiple sclerosis and Arn } \\
\text { malformation may manifest with hearing loss and verti }\end{array}$ \\
\hline \multicolumn{2}{|c|}{ Table 2 Epidemiological data } \\
\hline & Epidemiological data \\
\hline & Women \\
\hline & Age between 30 and 45 years \\
\hline ble 3 Clinical cl & aracteristics of hearing loss \\
\hline \multicolumn{2}{|c|}{ Clinical features of hearing loss } \\
\hline \multicolumn{2}{|c|}{ Usually affects severe frequencies } \\
\hline \multicolumn{2}{|l|}{ Asymmetric } \\
\hline \multicolumn{2}{|l|}{ Floating } \\
\hline \multicolumn{2}{|c|}{ Neurosensory } \\
\hline \multicolumn{2}{|c|}{ Rapidly progressive (weeks to months) or sudden } \\
\hline \multicolumn{2}{|c|}{ Unilateral or bilateral } \\
\hline Tinnitus, ear & fullness and vertigo \\
\hline
\end{tabular}
e. Ménière's disease
f. Autoimmune diseases
g. Viral diseases
h. Exposure to intense noises
i. Hypertension
j. Oto and sclerosis
k. Trauma in the head region
1. Tumors
m. Aging (which is one of the most common causes). ${ }^{2}$

\section{Clinical manifestations}

Associated with the syndrome or spontaneous mutation Hearing loss present at birth, progressive from birth or early adulthood. Hearing loss in varying degrees and may be the cause of profound congenital deafness.

More significant after 60 years, bilateral, symmetrical, beginning with loss in high frequencies (acute). Tinnitus may be associated.

Episodic seizures of vertigo Worsening of hearing loss and tinnitus during seizures, atrial fullness Hearing loss in low frequencies, fluctuating Hearing loss begins at high frequencies, with gradual progression.

Usually the hearing loss does not exceed $45 \mathrm{~dB}$ in the low frequencies and $75 \mathrm{~dB}$ in the high ones.

Unilateral or asymmetric hearing loss Tinnitus, unilateral, imbalance, headache, facial hyperesthesia.

Usually bilateral hearing loss, symmetrical and slowly progressive.

Bilateral, asymmetric, fluctuating, sudden or rapidly progressive hearing loss Tinnitus, vertigo and atrial fullness.

Hearing loss associated or not with tinnitus, peripheral labyrinth.

Stroke: hearing loss, ataxia of recent onset, dysarthria, vertigo, instability.Arnolda-Chiari: hearing loss, facial pain, headache, paresis.
The degree of hearing loss varies from person to person and is usually linked to:

a. Genetic inheritance

b. The abnormal conditions to which the ear was exposed during life

c. General disorders such as hypertension and diabetes, which may also affect the inner ear. ${ }^{2}$

d. Problems in the inner ear usually cause difficulty in separating speech from external noises, difficulty hearing high frequency sounds and the need to repeat what has been said. ${ }^{2}$

e. The early diagnosis is essential for treatment to be instituted as early as possible, aiming at a better end result. When deterioration of inner ear structures is noted, hearing loss is almost always irreversible. It is estimated that in about $95 \%$ of cases the use of hearing aids is effective in reducing the impact of the problem. In the case of severe to profound sensorineural hearing loss, the solution may be the cochlear implant (Table 5). ${ }^{5}$ 
Table 4 Requested examinations and results

\begin{tabular}{lll}
\hline & Exams & Results \\
\hline Non-specific & FAN, FR, Hemogram, Mucoproteins, HSV/PCR & If increased, they suggest the presence of autoimmune disease. \\
Specific & Anti-proteína antibody $68 \mathrm{kD}$ (Western Blot) & $\begin{array}{l}\text { Positivity suggests presence of DNSI. } \\
\text { Image }\end{array}$ \\
\hline
\end{tabular}

Table 5 Treatment

\begin{tabular}{lll}
\hline Corticoterapia & Immunosuppressants & Plasmapheresis \\
\hline Initial: I to $2 \mathrm{mg} / \mathrm{Kg} /$ day of prednisone, & Cyclophosphamide 2 to & 10 sessions for I month, in conjunction with \\
for 4 weeks. & $5 \mathrm{mg} / \mathrm{kg} /$ day; for non-responsive cases. & corticoid and cyclophosphamide; for cases still resistant. \\
Patients with good response should continue to receive high doses for another I or 2 months; \\
Later, the corticoid should be withdrawn gradually and decreasingly.
\end{tabular}

\section{Goals}

To identify the possible pathophysiological mechanisms involved in the involvement of the inner ear in autoimmune disease; and there was a protocol to be followed to conduct a suspected case of DNSI, from diagnosis, to treatment.

\section{Material and methods}

This research was a bibliographic review, exploratory, descriptive of qualitative method, involving articles published between the years of 2002 and 2014. The keywords used for electronic literary search involved combinations with the following words: hearing loss, autoimmunity, sensorineural hearing loss. In this step were selected bibliographies, selection of texts, websites, journal articles, with the purpose of gathering information related to the theme chosen for the work proposed here.

\section{Development}

Most cases of hearing loss in adults are sensorineural. This type of hearing loss affects the inner ear and / or the neural pathways. ${ }^{4}$ In this case, the sound is usually conducted through the outer ear and middle ear, but the inner ear presents less efficiency in the transmission of sounds, usually due to the reduction or damage to the nerve endings within the cochlea. This leads to a lower perception of the intensity and quality of the sound. The result is a deficiency in listening and understanding. ${ }^{4}$ Sensorineural hearing loss can be treated with hearing aids, the most frequent symptom is a sensorineural hearing loss, usually bilateral, progressive rapidamente and resposta varied to immunosuppressant's, since it depends on the degree of hearing loss. ${ }^{6}$

\section{Dysacusis immunomedial neurosensory- DNSI}

Immune- mediated sensorineural hearing loss-DNS I is classified as a form of acquired DNS, not late genetics and manifests as sensorineural, fluctuating, bilateral hypoacusis, rapidly progressive and responsive to treatment with immunosuppressive drugs. Approximately $65 \%$ of cases occur in women aged between 20 and $40 y e a r s$, being extremely rare in the pediatric population. ${ }^{4,5}$ in their studies characterized the clinical picture of immune-mediated sensorineural hearing loss-DNS I as sensorineural hearing loss, aural fullness symptoms such as tinnitus, and not tissue destruction or not the middle ear or external and pa and ralisia face. Sudden or progressive deafness, fluctuating and, uni-or bilateral, does not rule out the autoimmune etiology.

In many ways, the immunity of the labyrinth resembles that of the brain. The hetero-labyrinthine barrier separates the labyrinth from the bloodstream, maintaining the ionic characteristics of the cochlear environment. Immunoglobulins are present in the peri-lymph, at a ratio of $1 / 1,000$, similar to the level of cephalo-rachidial fluid. ${ }^{7}$

According Woolf ${ }^{8}$ the inner earseems to be moreimmunorresponsive than the brain, because the antigen present in the cochlea rapidly stimulates systemic immunity. When an antigen is introduced into the inner ear of previously sensitized animals, there is an immune response characterized by cellular infiltration, inflammatory reaction, cochlear damage, increase of antibodies in the perilymph, and elevation of local AC production. The inner ear is capable of presenting humoral immune response against a local or systemic antigenic stimulation; send to endolymphatic sac the area with the greatest potential to immune processing. ${ }^{9}$ According to Tomiyama ${ }^{10}$ the end lymphatic sac has a fixed population of lymphocytes, but curiously, the lymphocytes that appear in the inner ear during the immune response come from the bloodstream. The entry point of the white blood cells in the inner ear seems to be the spiral modiolar vein-VME which, in the cochlea, has a similar function to the post-capillary veins of other tissues. During the secondary immune response, the endothelial cells of the EMV are activated, assuming the characteristics of differentiated endothelial veins. These express intercellular adhesion molecules, recruiting circulating lymphocytes, with the likely contribution of other surface molecules.

The immune response of the inner ear is critical in protecting against infection; however, the associated inflammatory process can produce damage to the delicate cochlear tissue. In addition, there is evidence that cochlear tissue itself may be the target of autoimmune reaction. ${ }^{11}$ Cecatto et al. ${ }^{9}$ says that in the inner ear may be the site of organ-specific disease, or more commonly present in a disease that takes a systemic way. This autoimmune mechanism is capable of triggering DNSI by self-injury of the inner ear. The main autoimmune mechanisms involved in internal ear dysfunction include: immediate hypersensitivity, with production of IgE immuno-globulins against cochlear antigens, immunocomplex deposits in the vascular stria and spiral ligament, direct action of cytotoxic $\mathrm{T}$ cells in the cochlea and hypersensitivity delayed immunological reactivity mediated 
by type II collagen. These mechanisms can act complementarily and often simultaneously. The main histopathological findings are degeneration of the spiral ganglion, cortiorgan atrophy, vascular stria degeneration, endolymphatic duct precipitation and atrophy, presence of macrophages and precipitates in the endolymph, as well as perivascular infiltrate and deposition of circulating immune complexes in the vascular stria. ${ }^{9}$ Table 1 summarizes the main entities involved in the differential diagnosis of sensorineural hearing loss.

\section{Source ${ }^{12}$}

\section{Incidence}

The DNSI usually affects middle-aged women. A study by Hughes et al. ${ }^{13}$ showed a predominance of females $(65 \%)$ in relation to males (35\%). Harris et al. ${ }^{6}$ also observed, in a series of 279 patients, $63 \%$ of women. This fact agrees with the female prevalence found in other autoimmune pathologies of the organism.

\section{Diagnosis}

What is essential in the diagnosis of DNS I is the clinical history, since there are no definitive laboratory tests yet. Histopathological examination is not yet possible, since the inner ear is unavailable for tissue biopsy. ${ }^{14}$ A DNSI may initially present isolated otologic symptoms. But it is most commonly part of an immune-mediated systemic disease. Stephens et al (2009), through a clinical study, showed a high incidence of cochlear-vestibular symptoms (in 13 of 17 patients with ICD). And yet, studies that investigated risk groups for developing DNS did not attempt to correlate otological clinical history with their audiological research findings. ${ }^{2}$ QUPrev to the suspected DNSI, a complaint phase clinica without audiometric expression, it should be Initials air treatment with immunosuppressive corticosteró go or when exist go important clinical suspicion, can be with this reversal or stabilization $d$ the degenerative process of the inner ear still in an incipient stage, preventing irreversible deafness. Recovery is directly related to cochlear lesion, more specifically to hair cells. Once occurring death of these cells, or immunosuppression restore auditory function. In patients with defined cochlear lesions, we should consider the interruption of the progression of the condition satisfactory, which will allow eventual rehabilitation with a hearing aid. $^{2}$

\section{The tests}

In laboratory tests between $\mathrm{m}$ which can aid in the diagnosis of DNSI can cite complete blood count, erythrocyte sedimentation rate (ESR), C-reactive protein (CRP), anti-neutrophil cytoplasmic antibody assay (ANCA), anti-factor nucleus (FAN), rheumatoid factor $(\mathrm{RF})$ and mucoproteins. The increase in these markers is suggestive of autoimmune disease. ${ }^{15}$ The Ac-anti $68 \mathrm{kD}$ is a highly specific test for DNSI, using the Western B lot method against $68 \mathrm{kD}$ hsp-70 (anticochlea antibody evaluation), and its positivity is around $89 \%$ in the active phase of the disease. Approximately $70 \%$ of the patients who present the positive test have a good response to treatment with corticosteroids, compared to $15 \%$ in patients who present a negative test. $^{16}$

The anti-68kD (70hsp) anti-protein AC research is currently the most specific test in otoimmunity, although rarely performed because of its high cost and difficult access. ${ }^{16}$

Since lymphocyte transformation in Consist method that exposes mononuclear leukocytes prepared from the patient's blood, various dilutions of antigen. ${ }^{17}$ The evaluation (of the test is done through positive and negative controls. Lymphocytes are then examined from scintigraphy techniques and compared to controls by statistical evaluations (Table 6) ${ }^{17}$ Its predictive value is around $80 \%$ and is considered alongside Western blot, one of the most specific tests in the diagnosis of modern otoimmunology. ${ }^{17}$ The literature is controvers relative to the role of anti-type II collagen antibody. Some authors publish ram results revealed a large percentage of patients with idiopathic progressive DNS Bilateral with high titers of anti collagen II Ac, observing also correlation between high titers of Ac and good response to therapy with corticosteroids front. ${ }^{18}$

Table 6 Prognostic evaluation

\section{Corticosteroid test}

The criteria indicative of responsiveness to corticotherapy (Monthly monitoring with audiometry) are:

Improvement in tritonal mean for pure tone by $15 \mathrm{~dB}$ or more;

Increase in vocal discrimination by $20 \%$ or more;

Stabilization of hearing with complete resolution of vertigo.

Circulating immunocomplexes- $\mathrm{ICC}^{2}$ have an important role in vasculitis and glomerulonephritis, and has been frequently related to hearing loss. Evidence of this fact includes the detection of CHF in the blood and its deposition in the organs, resulting in tissue damage due to the activation of the complement cascade. ${ }^{17}$ The complement system-C1q is activated para there is precipitation of the ICC and tissue damage, there must be precipitation after consumption of specific complement fractions. ${ }^{17}$

In this sense, we investigated the fraction $\mathrm{C} 1 \mathrm{q}$, which has a special affinity for the immunocomplexes, being reduced in the blood levels of the patients with DNSI. ${ }^{17}$

Another test is the HLA. We have already reported the incidence of $51 \%$ of HLA Cw7 in patients with DNS I when compared to the control group. And studos point out the higher incidence of HLA B16 and B18 in carriers of DNS I, in addition to HLA DR2 and Cw4, much more frequently than in the rest of the population. ${ }^{19}$

Although it is recommended carrying out the tests mentioned often gets If no definitive diagnosis, as serum levels indicated for positivate exams are rarely achieved in the case of localized and limited process, as is the case of the endolymphatic sac. ${ }^{16}$ Thus, there is little relation between the positivity of the cited tests and the response to treatment. ${ }^{16}$

Thus, the clinical suspicion persist going DNSI, although no confirmation laboratory opts If the therapeutic test with corticosteroids ${ }^{3}$ as a means of confirming the initial diagnostic hypothesis. ${ }^{6}$ It is worth mentioning the use of imaging methods for diagnosis, such as Magnetic Nuclear Resonance (NMR) of temporal bones. We note the hypersignal cochlear, after infusion of paramagnetic contrast (gadolinium), in the images obtained in Table 1, when in the presence of inflammatory process. In cases of ICD, MRI may show other systemic changes of the central nervous system, such as vasculature of small vessels. ${ }^{15}$

${ }^{2} \mathrm{ICC}$ can be detected in plasma and electrodensing deposits in tissue through immunofluorescence. Its presence in the blood determines the suspected autoimmune process in progress.

${ }^{3}$ It consists of observing the type of audiometric response to the use of prednisone. The test is performed with the administration of prednisone at a dose of $1 \mathrm{mg} / \mathrm{kg} /$ day for a period of 15-20days. The positivity is given by the improvement of the tonal thresholds at the end of the test, which closes the autoimmune reaction diagnosis of the inner ear. 


\section{Treatment}

\section{The DNSI treatment is done by:}

Corticosteroid treatment: patients who have urges tion suggestive of immune-mediated cause hearing loss should undergo immunosuppressive therapy. Initial treatment should be performed using prednisone at a dose of 1 to $2 \mathrm{mg} / \mathrm{kg}$ /day for 4weeks. P TIENTS with good response should continue to receive high doses for 1 or 2 months, always with audiometric controls me NSAIs. Subsequently, corticoid should be withdrawn gradually and decreasingly. Du Rante administration of cortico ide, should monitor the side effects of corticosteroid therapy and the risk-benefit ratio should be fully discussed with the patient and their family members. ${ }^{16}$ The criteria indicative of responsiveness to corticotherapy are: 1) improvement in tritonal mean for pure tone by $15 \mathrm{~dB}$ or more; 2 ) increased vocal discrimination by $20 \%$ or more; and 3) stabilization of hearing with complete resolution of vertigo. ${ }^{2}$ The tes Patients may not respond to corticosteroid therapy with prednisone and/or prednisolone and may have liver dysfunction induced by these ides O Cork, Ireland. In these cases, one may choose to use betamethasone $20 \mathrm{mg} /$ day or dexamethasone $30 \mathrm{mg} /$ day. The side effects resulting from the use of corticosteroids are Cushing's syndrome, systemic arterial hypertension and diabetes, and the use of immunomodulators is therefore recommended. Contraindications to the use of ester $\mathrm{O}$ ides include: pregnancy, hypersensitivity to the drug, peptic ulcer disease, a history of tuberculosis, hypertension, glaucoma, diabetes, and other recent vaccination. ${ }^{16}$

Immunosuppressants: In $\mathrm{m}$ non-responsive cases, cytotoxic agents may be added. Cyclophosphamide should be used in the dose of 2 to $5 \mathrm{mg} / \mathrm{kg}$ / day in 1 daily intake in the morning, together with the intake of enough liquid, due to its nephrotoxicity. Administration of cyclophosphamide has been associated with the development of hemorrhagic cystitis and malignant diseases of the urinary tract. Under these conditions, methotrexate and azathioprine have been recommended. It is essential to inform the patient about the risks related to the treatment, which should be done with the help of a rheumatologist or hematologist/oncologist. ${ }^{16}$

Plasmapheresis: In still resistant cases, plasmapheresis should be considered daily for 5 days, followed by alternating days, for 2 weeks and 2 times per week, for a total of 10 sessions, for 1 month, when the results of the therapy will be re-observed. If the response is positive, the maintenance treatment can be done every 10days or so. During plasmapheresis, steroids and cyclophosphamide should be continued to enhance the immunosuppressive effect and prevent the continuous production of antibody. ${ }^{16}$

Are studied further the use of cortico go intratimp anode, this being a possible alternative for patients intolerant to oral systemic corticosteroids, such as those having uncontrolled hypertension or diabetes mellitus decompensated, or for patients with responsiveness to corticosteroids only in very high doses. The drug of choice is dexamethasone, with methylprednisolone as its second option. Reports in the literature differ in relation to the concentration of the solution (dexamethasone: $2-4 \mathrm{mg} / \mathrm{mL}$ at $25 \mathrm{mg} / \mathrm{mL}$; methylprednisolone $32 \mathrm{mg} /$ $\mathrm{mL}$ to $62.5 \mathrm{mg} / \mathrm{mL}$ ). The duration of treatment time, interval between injections and number of injections also differs between several authors ranging from a single dose, weekly transthyppanic injections, multiple weeks with solution (drops) of steroids administered by the patient himself, transthyppanic injections administered several times by week or through an implantable infusion pump. ${ }^{20}$
Different minds systemic therapies, intratympanic therapy allows direct and specific action in the ear afetada being treated. In addition to glucose intolerance and a vascular necrosis of the hip, other adverse effects of systemic corticosteroids such as insomnia, irritability, gastritis and mood changes can be avoided through intratympanic therapy. The main disadvantage of intratympanic corticosteroid therapy is still the lack of evidence that it would have a superior efficacy when compared to systemic therapy. Other disadvantages include pain, vertigo and even rare possibility of perforation of the tympanic membrane and serous otitis media. ${ }^{20}$ In studies effect was not observed with the ADD al concomitant oral corticoid of the anode and intratimp. ${ }^{20-26}$

\section{Conclusion}

It can be concluded that the sensorineural hearing loss may occur in patients with autoimmune disease and should always be remembered in cases of discuses with no apparent cause. Knowledge of autoimmune diseases and their correlation with the picture of disabuse sensorineural a major contributor in the demystification of this type of hearing loss allowing specific treatment institution more precocem one, with better results. The main autoimmune mechanisms involved in internal ear dysfunction include: immediate hypersensitivity, with production of IgE immuno- globulins against cochlear antigens, immunocomplex deposits in the vascular stria and spiral ligament, direct action of cytotoxic $\mathrm{T}$ cells in the cochlea and hypersensitivity delayed immunological reactivity mediated by type II collagen. These mechanisms can act complementarily and often simultaneously.

\section{Acknowledgments}

None.

\section{Conflicts of interest}

The author declares there is no conflict of interest.

\section{References}

1. Decoster DMH, Ferreira NGM, Marques MPC. Autoimmune diseases of the inner ear-a bibliographic review. Acta Awho. 2010.

2. Cruz OLM, Alvarenga EL, Costa SS. Clinical and surgical Otology $11^{\text {th }}$ edition, Revinter; 2008 .

3. Hirose K, Wener MH, Duckert LG. Utility of laboratory testing in autoimmune inner ear disease. Laryngoscope. 1999;109(11):17491754.

4. Veldman JE. Immune-mediated sensorineural hearing loss with or without endolynophatic hydrops: a clinical and experimental approach. Ann N Y Acad Sci. 1997;830:179-186.

5. Mccabe BF. Autoimmune sensorineural hearing loss. Ann Otol Rhinol Laryngol. 2007;116(12):875-879.

6. Harris JP. Immunologic Disorders Affecting the ear. Cummings C. Otolaryngology Head and Neck Surgery. United States; 2009.

7. Mogi G, Lim DJ, Watanabe N. Immunologic study on the inner ear: immunoglobulins in perilymph. Arch Otolaryngol. 1982;108(5):270275.

8. Woolf NK, Harris JP. Cochlear pathophysiology associated with inner ear immune responses. Acta Otolaryngol. 1986;102(5-6):353-364.

9. Cecatto, Suzana B. et al. Sensorineural hearing loss in Systemic Lupus Erythematosus: report of three cases. Brazilian Journal of Otorhinolaryngology. 2004;70(3):398-403. 
10. Tomiyama S, Harris JP. The role of the endolymphatic sac inner ear immunity. Acta Otolaryngol. 1987;103(5-6):182-188.

11. Suzuki M, Harris JP. Expression of intercellular adhesion molecule-1 in the inner ear during experimental labyrinthitis in rat. Ann Otol Rhinol Laryngol. 2009;104:69-75.

12. Lavinsky-Wolff M, Lavinsky L. Comparative analysis of two cochlear implant techniques: cohort study with long-term outcome [dissertation]. Porto Alegre: Federal University of Rio Grande do Sul; 2012.

13. Hughes GB, Kinney SE, Barna BP, et al. Practical versus theoretical management of autoimmune inner ear disease. Laryngoscope. 1984;94(6):758-767.

14. Bowman CA, Nelson RA. Human leukocytic antigens in autoimmune sensorineural hearing loss. Laryngoscope. 1987;97(1):7-9.

15. Penido Norma O. Immune-mediated sensorineural dysacusia. Brazilian Journal of Otorhinolaryngology. 2002;68(5):730-734.

16. Moscicki R. Western blot analysis of serum antibody to inner ear antigens in patients with idiopathic progressive bilateral sensorineural hearing loss (IPBSNHL). American Neurotology Society Meeting. 2010;27-29.

17. Paleri V. Inner ear immunology and allergy: In the overview of current day concepts. Ear Nose Throat J. 1997;76(11):799-802.

18. Berger P, Koja S, Rogowski M, et al. The lynphocyte transformation test for detecting immunologic inner ear deafness. HNO. 1989;37(4):153157.
19. Koyama S, Mitsuishi Y, Bibee K. HLA Associations with Meniere's Disease. Acta Otolaryngol. 1993;113:575-578.

20. Raymundo IT, Bahmad F Jr, Barros Filho J, et al. Intratympanic methylprednisolone as rescue therapy in sudden sensorineural deafness. Braz J Otorhinolaryngol. 2010;76(4):499-509.

21. Abbas Abul K, Lichtman Andrew H, Pillai Shiv. In: Cellular and molecular immunology. Philadelphia: Saunders; 2011.

22. Dinces EA, Yang SAB, Balogun AO. Pediatric fluctuating sensorineural Hearing Loss: Problems in medical management. Laryngoscope. 2001;111(1):21-25.

23. Arnold W, Pfaltz CR. Critical evaluation of immunofluorescence microscopy for the identification of serum antibodies against human inner ear tissue. Acta Otolaryngol. 1987;103(5-6):373-378.

24. Richardson Roberto J. Social Research: methods and techniques. $3^{\text {rd }}$ edition. São Paulo: Atlas; 2009.

25. Stephens SD, Luxon L, Hinchcliffe R. Immunological disorders and auditory lesions. Audiology. 1982;21(2):128-148.

26. Triviños ANS. Introduction to Research in Social Sciences: Qualitative Research. São Paulo: Atlas; 2006. 